\title{
Outer spiral structure in disk galaxies
}

\author{
P.A. Patsis \\ Research Center for Astronomy, Academy of Athens \\ Soranou Efessiou 4, GR-11527, Athens, Greece \\ email: patsis@academyofathens.gr
}

\begin{abstract}
In several grand design barred-spiral galaxies it is observed a second, fainter, outer set of spiral arms. Typical examples of objects of this morphology can be considered NGC 1566 and NGC 5248. I suggest that such an overall structure can be the result of two dynamical mechanisms acting in the disc. The bar and both spiral systems rotate with the same pattern speed. The inner spiral is reinforced by regular orbits trapped around the stable, elliptical, periodic orbits of the central family, while the outer system of spiral arms is supported by chaotic orbits. Chaotic orbits are also responsible for a rhomboidal area surrounding the inner barred-spiral region. In general there is a discontinuity between the two spiral structures at the corotation region.
\end{abstract}

Keywords. galaxies: structure, galaxies: kinematics and dynamics

\section{A grand design morphology with two sets of spirals}

Stellar and gas response models indicate that in barred-spiral models with a single pattern speed and with corotation to bar radius $R_{c} / R_{b} \approx 2.9$ a morphology as the one depicted in Fig. 1 is established after 2-3 pattern rotations.

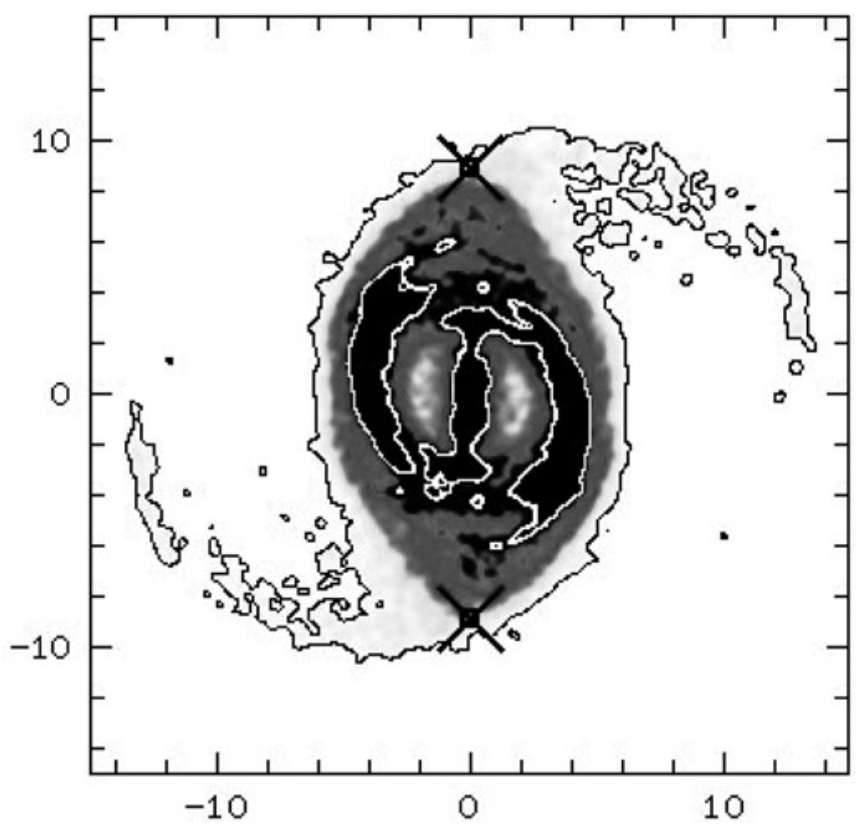

Figure 1. A stellar barred-spiral response model with two sets of spirals. " $\times$ " symbols indicate the location of the unstable Lagrangian points. 